\title{
Disparate Tendency of Stress Evolution of Thin and Thick Electroplated Cu Films at Room Temperature
}

\author{
Rui Huang ${ }^{\mathrm{a}, \mathrm{b}}$, Werner Robl ${ }^{\mathrm{c}}$, Gerhard Dehm ${ }^{\mathrm{d}, \mathrm{e}}$, Hajdin Ceric ${ }^{\mathrm{b}}$, Thomas Detzel $^{\mathrm{f}}$ \\ ${ }^{a}$ Kompetenzzentrum Automobil-und Industrieelektronik (KAI) GmbH, Europastrasse 8, 9500 Villach, Austria \\ ${ }^{\mathrm{b}}$ Institute for Microelectronics, TU Wien, Gusshausstrasse 27-29, 1040 Vienna, Austria \\ 'Infineon Technologies AG, Wernerwerkstrasse 2, 93049 Regensburg, Germany \\ ${ }^{\mathrm{d} D e p a r t m e n t}$ of Materials Physics, Montanuniversität Leoben, Jahnstrasse 12, 8700 Leoben, Austria \\ ${ }^{\mathrm{e}}$ Erich Schmid Institute of Material Science, Austrian Academy of Sciences, Jahnstrasse 12, 8700 Leoben, Austria \\ Infineon Technologies Austria AG, Siemensstrasse 2, 9500 Villach, Austria \\ Phone: (+43)4242-34890-16 Fax: (+43)4242-34890-99 Email: rui.huang@k-ai.at
}

\begin{abstract}
The self-annealing effect of electroplated copper films was investigated by measuring the time dependence of the film stress for different film thicknesses between $1.5 \mu \mathrm{m}$ and $20 \mu \mathrm{m}$. This recrystallization process results in significant microstructure change and a film-thickness-dependent stress evolution at room temperature. This behavior can be explained by the superposition of grain growth and grain size dependent yielding. Existing models have been used and improved to describe the mechanisms related to stress evolution.
\end{abstract}

\section{INTRODUCTION}

Copper becomes more and more important for interconnect applications in semiconductor devices due to its higher electrical conductivity, increased electro-migration resistance and better thermal conductivity $[1,2]$. Electroplating of copper has been demonstrated as one of the best methods to be adopted for high performance logic devices using dual damascene technology and for power devices using pattern plating technology [3, 4]. Recrystallization of electroplated $\mathrm{Cu}$ films at room temperature, which is termed self-annealing, is a very distinct phenomenon [5-9]. During self-annealing, stress change is observed, which is associated with a transition from an as-deposited ultrafine-grained microstructure to a coarse-grained microstructure. The self-annealing impacting subsequent processing steps may require expensive post deposition treatments and can cause reliability impairment. Therefore, a fundamental understanding of the self-annealing phenomenon is of great scientific and technological importance in order to ensure the stability of electroplated $\mathrm{Cu}$ metallization.

So far most of the research has been devoted to electroplated $\mathrm{Cu}$ films with thicknesses ranging from several hundred nanometers up to about $2 \mu \mathrm{m}$ [10-19], but was recently extended to studies on $70 \mu \mathrm{m}$ thick $\mathrm{Cu}$ films $[20,21]$. In these studies, grain growth was observed during room temperature annealing, accompanied by a continuous relaxation of film stress. The grain growth is believed to be either induced by the initial high dislocation density triggering recrystallization or by surface segregation of impurities releasing the pinned grain boundaries.
If the stress is initially compressive, grain growth and annealing of defects will cause a reduction of the stress. However, for films which are initially under tensile stress after deposition, grain growth would increase the tensile stress and therefore can't explain a stress decrease. In such cases other mechanisms must be active, which will be discussed in this paper. Besides that, only very few publications can be found which present the modeling results regarding the self-annealing effect. Still, most of them only focus on the modeling in terms of sheet resistivity evolution $[22,23]$. The purpose of the present work is also to develop a model which is able to describe the mechanisms of stress evolution of electroplated $\mathrm{Cu}$ films so that the prediction of the stress evolution at room temperature becomes feasible.

\section{EXPERIMENTAL AND RESULTS}

All investigated $\mathrm{Cu}$ films were prepared by electroplating on 8 inch $(\sim 200 \mathrm{~mm})$ blanket wafers. Prior to the electroplating, a $100 \mathrm{~nm}$ thick thermal oxide was deposited by chemical vapor deposition (CVD) on the wafers as an isolation layer. Subsequently, a 50nm thick $\mathrm{Ti}$ film was deposited as an adhesion layer and diffusion barrier, followed by a 150nm thick $\mathrm{Cu}$ sputter deposited seedlayer. The barrier layer and seedlayer were deposited in the same sputter tool without breaking the vacuum. The plating process was performed with a commercial fountain plating system. The electrolyte solution was mainly composed of $\mathrm{Cu} 2 \mathrm{SO} 4, \mathrm{H} 2 \mathrm{SO} 4, \mathrm{HCl}$ and with two organic additives, an accelerator and a suppressor with levelling components. An AC-DC current combination was applied [24].

The whole electroplating process consecutively undergoes a pre-wet in methane sulphonic acid (MSA), $\mathrm{Cu}$ electroplating and a water rinse followed by a spin-dry process. The plating rate was kept constant for all $\mathrm{Cu}$ thicknesses, so that the impact of deposition speed on film properties was avoided. Finally, a wide split plan of $\mathrm{Cu}$ thickness was applied from 1.5-20 $\mu$ m.All blanket electroplated $\mathrm{Cu}$ layers were characterized as a function of time at room temperature. In order to determine the film 
stress, the wafer curvature was monitored by a capacitance probe setup (Eichhorn\&Hausmann) starting 30min after deposition up to $\sim 100 \mathrm{~h}$, based on which film stress can be calculated by using Stoney's equation [25-27]:

$$
\sigma=\frac{E h^{2}}{(1-v) 6 t}\left(\frac{1}{R_{2}}-\frac{1}{R_{1}}\right)
$$

where $\sigma$ is the film stress, $E$ corresponds to the elastic modulus of the substrate, $v$ denotes the Poisson's ratio of the substrate, $h$ and $t$ are the thickness of the substrate and film, $R_{l}$ and $R_{2}$ are the measured radii of curvature before and after $\mathrm{Cu}$ film electroplating, respectively. Thus, for all reported stresses of electroplated $\mathrm{Cu}$ films contributions from the underlying thermal oxide layer as well as the $\mathrm{Ti}$ and $\mathrm{Cu}$ seedlayers have been eliminated.

As illustrated in Fig.1, all films show a tensile stress. Thin $\mathrm{Cu}$ films $(1.5-5 \mu \mathrm{m})$ have relatively high initial stress compared to thick $\mathrm{Cu}$ films $(8-20 \mu \mathrm{m})$. The stress evolution shows a disparate tendency between these two groups: i) thin films with the thickness of $5 \mu \mathrm{m}$ and below, ii) thick films with the thickness of $8-20 \mu \mathrm{m}$. The tensile stress of thin films continues to decrease with time, while for thick $\mathrm{Cu}$ films the stress first increases and subsequently starts to decrease or stagnate depending on the maximum stress value. Finally, the tensile stress values of all $\mathrm{Cu}$ films stagnate at a certain value, which is determined by the film thickness. Thicker films reveal a lower final stress than thinner films. Interestingly, the as-deposited film stress with its nanocrystalline microstructure shows also an inverse relationship between film stress and film thickness.

The microstructure evolution was investigated for $3 \mu \mathrm{m}, 8 \mu \mathrm{m}$ and $20 \mu \mathrm{m}$ thick films $2 \mathrm{~h}, 20 \mathrm{~h}$ and $44 \mathrm{~h}$ after deposition by focused ion beam (FIB) microscopy (Micrion 9500). The average grain size is calculated by a linear intercept method based on FIB images recorded at $10 \mathrm{~K}$ magnification. The grains on cross-section from bottom to top excluding the extreme top ones at the edge were captured to estimate the grain size. Twins are counted as grains. Due to the resolution limit of FIB images, the minimum grain size that can be detected is $\sim 50 \mathrm{~nm}$. The $45^{\circ}$ tilt angle of the FIB images has been taken into account for the grain size analysis in this study.

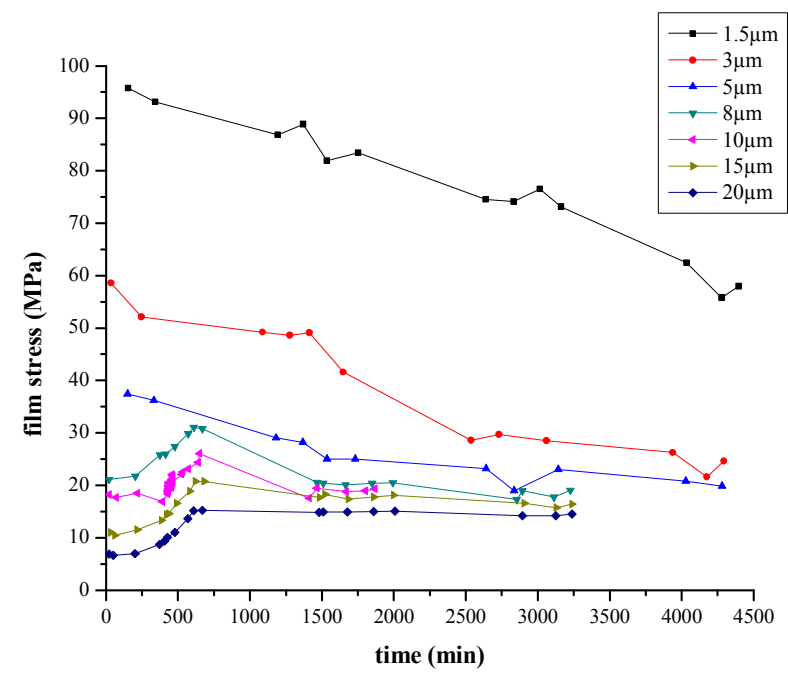

Fig.1. Stress evolution of $1.5-20 \mu \mathrm{m}$ thick $\mathrm{Cu}$ films at room temperature. Disparate tendencies of stress are observed between thin $\mathrm{Cu}$ films $(1.5-5 \mu \mathrm{m})$ and thick $\mathrm{Cu}$ films $(8-20 \mu \mathrm{m})$.

The images in Fig.2 made by FIB technique show a remarkable grain growth of $8 \mu \mathrm{m}$ thick $\mathrm{Cu}$ film during self-annealing. The first FIB image taken $2 \mathrm{~h}$ after deposition reveals a fine globular grain structure. After 20h, a significant change in grain size has occurred. The grains which frequently contain twins have a bimodal grain size distribution. It should be noted, that at the $\mathrm{Cu} /$ substrate interface, a $\sim 300 \mathrm{~nm}$ thick region of fine grains still exists. After $44 \mathrm{~h}$, all fine grains have been consumed by large grains. Some of the grains (columnar grains) extend through the complete thickness of the film and contain twins. The coexistence of columnar grains and twins leads again to a bimodal grain size distribution. The average grain sizes of $8 \mu \mathrm{m}$ thick $\mathrm{Cu}$ film are approximately $135 \mathrm{~nm}, 210 \mathrm{~nm}$ and $290 \mathrm{~nm}$ according to the FIB images taken after $2 \mathrm{~h}, 20 \mathrm{~h}$ and $44 \mathrm{~h}$ at room temperature, respectively.

\section{DISCUSSIONS}

\section{A. mechanisms of stress evolution}

Two different film thickness regimes are identified from the stress-time curves. Therefore, both regimes will be discussed and possible mechanisms for the different behaviors will be

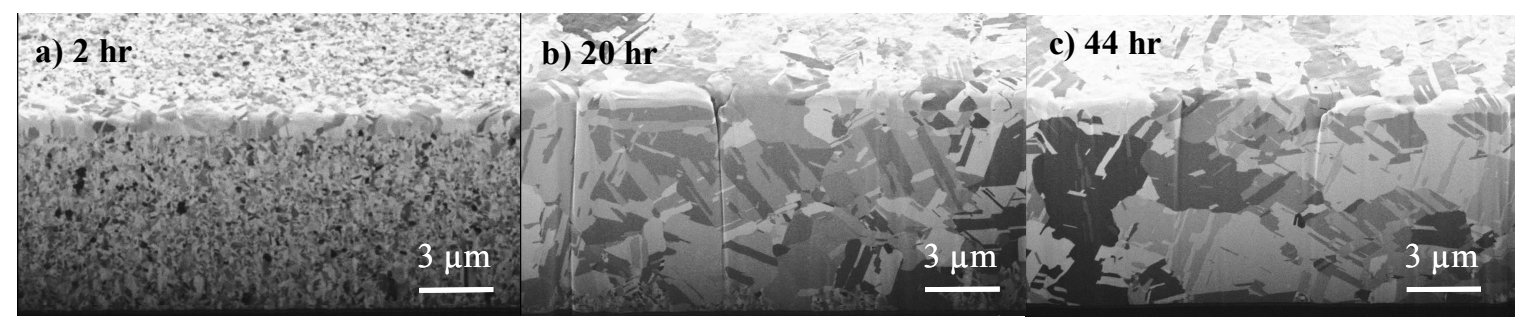

Fig. 2. Microstructural evolution of $8 \mu \mathrm{m}$ thick $\mathrm{Cu}$ film at room temperature. a). 2 hours after deposition only fine grains are existing in the film; b). 20 hours after deposition coarse grains are formed while fine grains are still existing at the bottom of the film; c). 44 hours after deposition, coarse grains occupy the film, where twins are embedded. 
proposed.

The continuous decrease of the stress of thin $\mathrm{Cu}$ films $(1.5 \mu \mathrm{m}-5 \mu \mathrm{m})$ in Fig. 1 cannot be directly explained by grain growth. It is speculated that dislocation plasticity causes the stress decrease, as outlined below. As the initial stress is relatively high for thin $\mathrm{Cu}$ films, dislocation glide can be activated immediately after deposition and becomes increasingly easier due to grain growth. The stress decrease terminates when the flow stress of the final microstructure, which is film thickness dependent, has been reached. The stress evolution of thick $\mathrm{Cu}$ films $(8 \mu \mathrm{m}-20 \mu \mathrm{m})$ is more complex. As the initial stress is relatively low and the grain size is small, dislocation glide cannot be activated immediately after deposition. Then grain growth starts causing a stress increase after the $\sim 200 \mathrm{~min}$ incubation phase for the $8 \mu \mathrm{m}-20 \mu \mathrm{m}$ thick $\mathrm{Cu}$ films. Grain growth leads to annihilation of excess volume by reducing the amount of grain boundaries which induces shrinkage of the film. This gives rise to the tensile stress if the film remains bonded to the substrate. As shown in the FIB images of Fig.2, grain growth clearly occurs during self-annealing. The coarsening starts with the growth of a few grains which consume the surrounding fine-grained matrix until the large grains meet and the fine-grained matrix is completely consumed. Along with grain growth, the tensile film stress increases until it becomes high enough to activate plasticity yielding. As a consequence, both grain growth and plasticity superimpose in the stress evolution. The global stress decreases when the effect of dislocation plasticity becomes more prominent than that of grain growth. This is the case for $8 \mu \mathrm{m}$ and $10 \mu \mathrm{m}$ thick $\mathrm{Cu}$ films. For the $15 \mu \mathrm{m}$ and $20 \mu \mathrm{m}$ thick films the peak stresses are so low that dislocation glide and grain growth counterbalance each other. One should be aware that grain growth also happens for the $1.5-5 \mu \mathrm{m}$ thick $\mathrm{Cu}$ films. However, no stress increase is observed during room temperature annealing since the initial stress is so high that relaxation by dislocation plasticity is more prominent than the stress increase induced by grain growth.

Fig.3 illustrates these processes. The as-deposited films are defect rich caused by the low deposition temperature process and $\mathrm{Cl}, \mathrm{S}$ and $\mathrm{C}$ impurities [16] coming from the electrolyte bath. The incubation time may be caused by the segregation of impurities via the grain boundaries to the film surface and/or film/substrate interface as sketched in Fig.3a and $3 \mathrm{~b}$. Then, Ostwald ripening $[6,28]$ can set in with grain growth starting at grains where grain boundaries are released first by surface and interface segregation of impurities (Fig3.a-c). It implies that the stress itself is not the only driving force for self-annealing. Otherwise the thicker film wouldn't anneal so rapidly. The driving force for room temperature grain growth originates from the minimization of the total energy of the system. A large variety of energies, such as strain energy, grain boundary energy, surface/interface energy, dislocation energy and stacking faults energy are assumed to be involved in this process. The existence of a high density of defects, mostly impurities, dislocations and grain boundaries introduced by room temperature electroplating, is considered as the main a)

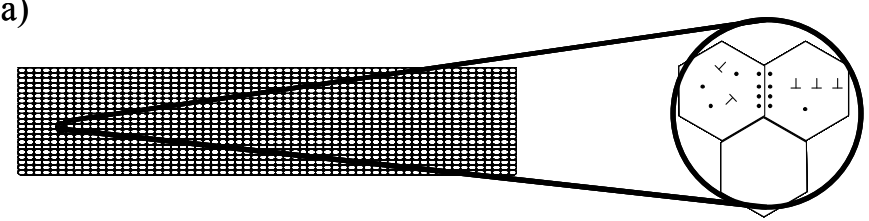

b)

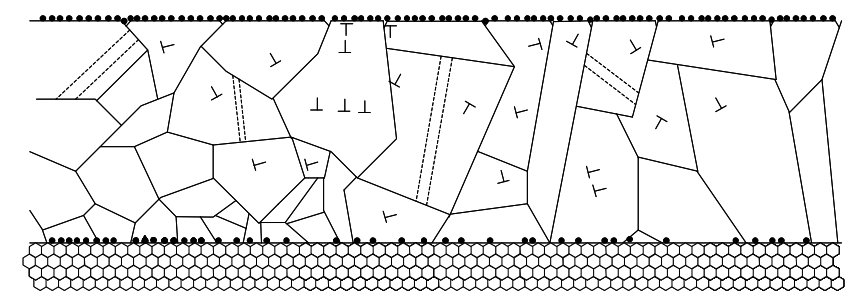

c)

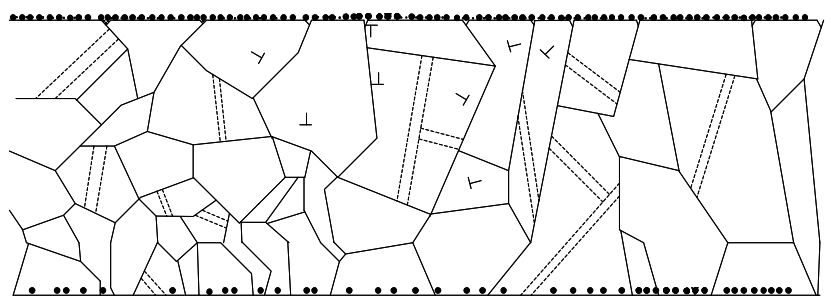

Fig.3. Illustration of the microstructure evolution. Dashed lines indicate twins. a) Only fine grains exist in the film after deposition, coupled with a high density of impurities and dislocations. Impurities pin at the grain boundaries; b) Impurities segregate to the film surface and/or film/substrate interface, thus releasing the grain boundaries. Grain growth starts and finally reaches the film/substrate interface region. Meanwhile, dislocation glide can be activated; c) Grain growth terminates. Dislocation density is reduced because dislocations glide across the grains and are incorporated into the grain boundaries.

source of a large amount of energy stored in the as-deposited fine grains of electroplated $\mathrm{Cu}$ films.

\section{B. modeling of stress evolution}

The loss of grain boundary volume due to grain growth induces the shrinkage of the film volume. If the film remains constrained to the substrate, it causes stress increase in tension. Chaudhari [29] has presented a model which explains the stress development due to grain growth. It calculates the strain in the film by estimating the change in volume associated with the coalescence of grain boundaries.

Considering a thin film that has an average grain size $L_{0}$ in the as-deposited condition, and assuming the grains grow to final grain size $L$, the total change of biaxial stress associated with grain growth is given by 


$$
\Delta \sigma_{g g}=\frac{E}{(1-v)} \frac{\alpha a}{2}\left(\frac{1}{L_{0}}-\frac{1}{L}\right)
$$

where $\Delta \sigma_{g g}$ is the stress increase induced by grain growth, $E$ is the elastic modulus of the film, $v$ is the Poisson's ratio of the film, $\alpha$ is a grain boundary parameter and $a$ is the bulk atom layer spacing.

On the other hand, grain boundaries as well as dislocations act as obstacles to dislocation motion at room temperature. Impeding the dislocation motion will hinder the onset of plasticity and hence increase the yield strength of the film. The dislocation pileup which determines the stress concentration in the grains, relates to the grain diameter and dislocation density. If the grain size is large and dislocation density is high, a greater stress concentration is developed in the grains, and thus the applied stress needed to activate plastic flow in the grains is relatively low, and vice versa. Hall-Petch law well represents the grain boundary barrier to dislocation motion as function of grain size [30-32]. By the modified Hall-Petch equation the stress decrease due to dislocation motion can be calculated:

$$
\Delta \sigma_{g b}=\sigma_{L}-\sigma_{L_{0}}=K\left(\frac{1}{\sqrt{L}}-\frac{1}{\sqrt{L_{0}}}\right)
$$

where $\Delta \sigma_{g b}$ is the stress change due to dislocation glide, $\sigma_{L}$ and $\sigma_{L_{0}}$ are the flow stress of the film in the case of the grain size of $L$ and $L_{0}$, respectively, $K$ is the Hall-Petch coefficient.

Furthermore, it additionally needs an intermediate model which introduces the grain size development as function of time elapsed so that the stress evolution can be correlated with time. Doerner and Nix [25] have successfully developed such a grain growth model to describe the rate of grain size development. This model is based on the minimization of grain boundary energy and strain energy.

$$
\frac{d L}{d t}=\frac{D^{*} \Omega}{k T \delta} \frac{2 \gamma}{L}\left(1-\left(\frac{E}{1-v}\right) \frac{(\alpha a)^{2}}{2 \beta \gamma}\left(\frac{1}{L_{0}}-\frac{1}{L}\right)\right)
$$

where $D^{*}$ is the diffusivity given by $D_{0} \exp \left(-E_{a} / k T\right), D_{0}$ is the pre-exponential factor for grain boundary diffusion, $E_{a}$ is the activation energy of grain boundary diffusion, $\Omega$ is the atomic volume, $\delta$ is the average jump distance in the grain boundary, $k$ is the Bolzmann constant, and $T$ is the absolute temperature, $\gamma$ is the grain boundary energy per unit area, $\mathrm{L}$ is the average grain size, and $\beta$ is the geometrical factor determined by the shape of the grains.

As aforementioned, the individual mechanism of grain growth or dislocation plasticity does not enable us to interpret the complex stress evolution of electroplated $\mathrm{Cu}$ films. Instead, a competing model combining the above two mechanisms, as shown in (5), needs to be applied in combination with grain growth rate model (4).

$$
\sigma=\sigma_{0}+\Delta \sigma_{g g}+\Delta \sigma_{g b}
$$

where $\sigma$ is the film stress and $\sigma_{0}$ is the as-deposited stress.

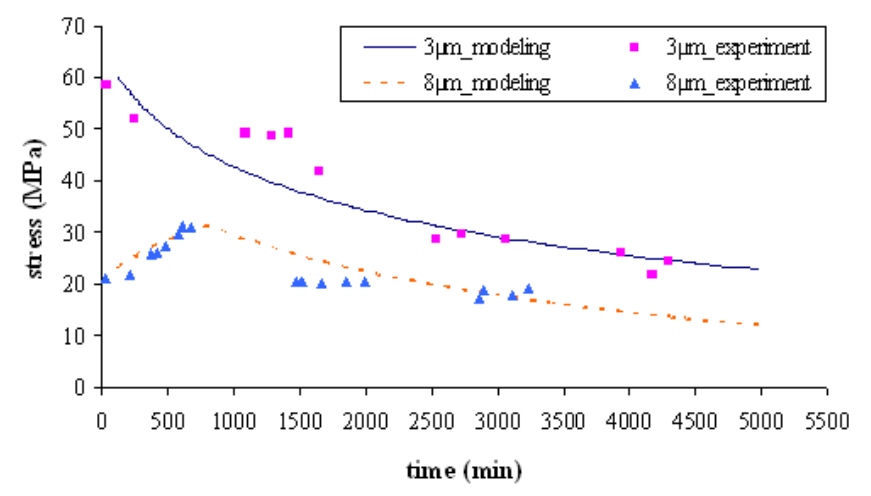

Fig.4. Experimental and modeled stress evolution of 3 and $8 \mu \mathrm{m}$ thick electroplated $\mathrm{Cu}$ films

Table 1. Parameters used in the modeling

\begin{tabular}{ccc}
\hline Symbol & Value & reference \\
\hline$\alpha$ & 0.12 & {$[6]$} \\
$a$ & $0.36148 \mathrm{~nm}$ & {$[6]$} \\
$\beta$ & 2 & {$[29]$} \\
$E$ & $121 \mathrm{GPa}$ & {$[6]$} \\
$\nu$ & 0.33 & {$[6]$} \\
$K$ & $0.0448 \mathrm{MN} / \mathrm{m}^{3 / 2}$ & {$[30]$} \\
$D_{0}$ & $3.04 \times 10^{-5} \mathrm{~m}^{2} / \mathrm{s}$ & {$[19]$} \\
$E_{a}$ & $1.46 \times 10^{-19} \mathrm{~J}$ & {$[19]$} \\
$\delta$ & $0.40486 \mathrm{~nm}^{-29}$ & {$[6]$} \\
$\Omega$ & $1.182 \times 10^{-2} \mathrm{~m}^{3}$ & {$[19]$} \\
$\gamma$ & $0.625 \mathrm{~J} / \mathrm{m}^{2}$ & {$[29]$} \\
\hline
\end{tabular}

Fig.4 shows the results of two representative numerical calculations in comparison with the corresponding experimental data labeled as dots. The as-deposited stress $\sigma_{0}$ is $60 \mathrm{MPa}$ and $23 \mathrm{MPa}$ for $3 \mu \mathrm{m}$ and $8 \mu \mathrm{m}$ thick $\mathrm{Cu}$ films, respectively. The grain sizes of 105 and $135 \mathrm{~nm}$ accordingly obtained from FIB images of 3 and $8 \mu \mathrm{m}$ thick $\mathrm{Cu}$ films are applied as original grain size $L_{0}$ in the simulation. In Fig.4, both calculated curves are qualitatively similar to the measured stress plots. As dislocation glide is prominent in the $\mathrm{Cu}$ films $(1.5-5 \mu \mathrm{m})$, continuous decrease of stress, which is fully represented by the calculated curve of $3 \mu \mathrm{m}$ thick $\mathrm{Cu}$ films, can be obtained by the described model with competing mechanisms. Meanwhile, the curve of $8 \mu \mathrm{m}$ thick $\mathrm{Cu}$ films is well on the behalf of thicker films $(8-20 \mu \mathrm{m})$, which conforms to grain growth and successive dislocation glide mechanisms.

It must be pointed out that the value of the grain boundary parameter $\alpha$ can vary with the deposition conditions, such as electrolyte composition or current density [33]. Moreover, grain growth and coalescence of grain boundaries are not homogeneous over the film, as seen in Fig. 2. It is very difficult to set the global $\alpha$ value accurately based on the actual local values over the film. In our model, it is assumed that $\alpha$ is the average over transformed and untransformed grains and it is estimated to be 0.12 based on the best fitting, which is fairly 
close to the value of 0.15 found by Cabral [34].

The Hall-Petch coefficient $K$ reflects the resistance of grain boundaries to dislocation motion. Typically, a lower value of $K$ refers to less resistance of dislocation motion, and vice versa. In Fig. 4, the modeled curves are calculated with the $K$ value of $0.0448 \mathrm{MN} / \mathrm{m} 3 / 2$, which is $\sim 40 \%$ of the well-known Hall-Petch constant $0.112 \mathrm{MN} / \mathrm{m} 3 / 2$ for face-centered cubic (FCC) copper [30]. During the abnormal grain growth, subgrains with low angle grain boundaries $\left(<15^{\circ}\right)$ may be strongly involved in the coarse-grained structure, which weakens the blocking of dislocations compared to high angle grain boundaries $\left(>15^{\circ} \mathrm{C}\right)$. Consequently, Hall-Petch coefficient related to subgrains is typically $1 / 2$ to $1 / 5$ of the well-known value. In order to capture the process of subgrain coarsening, in-situ electron backscatter diffraction (EBSD) or transmission electron microscopy (TEM) during grain growth would be required.

There is a notable limitation of the model, which is associated with incubation of grain growth. As observed in Fig.1, particularly in the stress evolution curves of thick $\mathrm{Cu}$ films $(8-20 \mu \mathrm{m})$, stress increase does not start immediately after deposition, but at $\sim 200 \mathrm{~min}$ after the deposition. This implies that the onset of grain growth is retarded and all fine grains maintain as-deposited undergoing an incubation phase. The incubation of grain growth is not considered in the present model. Thus, the simulated results only emphasize on the stress evolution after the commencement of grain growth, but neglect the stress stabilization during incubation. Nevertheless, compared to the total evolution time, the incubation time is relatively short.

\section{CONCLUSIONS}

New insights have been gained in the stress evolution during self-annealing of electroplated $\mathrm{Cu}$ by studying film thicknesses between $1.5 \mu \mathrm{m}$ and $20 \mu \mathrm{m}$. Grain growth is observed by focused ion beam studies. This is explained by segregation of impurities towards the film surface and interface unpinning the grain boundaries. For thick films the low as-deposited tensile stress increases due to annihilation of excess volume, while for thin films due to the already pre-existing high as-deposited tensile stress grain growth induces yielding and thus a reduction in film stress. Finally, grain growth terminates and stress relaxation by dislocation glide is exhausted. The film stress stagnates at a certain value which scales with film thickness.

The present study reveals that the stress evolution after deposition of electroplated copper films can be explained by grain growth and grain size dependent yielding. Quantitative models with competing mechanisms have been developed to simulate the stress evolution of electroplated $\mathrm{Cu}$ films. The results show an accurate correlation between experimental data and simulated values. To extend the model, incubation phase of grain growth may be considered. Further, EBSD or TEM for the investigation of subgrains is required in order to determine important model parameters, such as the Hall-Petch coefficient.

\section{ACKNOWLEDGMENT}

The authors would like to thank M. Roemet for focused ion beam measurements. Furthermore, M. Schneegans and P. Nelle are gratefully acknowledged for helpful discussions. This work was jointly funded by the Federal Ministry of Economics and Labor of the Republic of Austria (contract 98.362/0112-C1/10/2005 and the Carinthian Economic Promotion Fund (KWF) (contract 18911 | 13628 | 19100).

\section{REFERENCES}

[1] C. K. Hu, B. Luther, F. B. Kaufman, J. Hummel, C. Uzoh, and D. J. Pearson, "Copper interconnection integration and reliability," Thin Solid Films vol. 262, pp. 84-92, 1995.

[2] M. D. Thouless, J. Gupta, and J. M. E. Harper, "Stress Development and Relaxation in Copper-Films During Thermal Cycling," Journal of Materials Research, vol. 8, pp. 1845-1852, Aug 1993.

[3] P. C. Andricacos, C. Uzoh, J. O. Dukovic, J. Horkans, and H. Deligianni, "Damascene copper electroplating for chip interconnections," IBM JOURNAL OF RESEARCH AND DEVELOPMENT, vol. 42, pp. 567-574, 1998.

[4] L. T. Romankiw, "A path: from electroplating through lithographic masks in electronics to LIGA in MEMS," Electrochimica Acta, vol. 42, pp. 2985-3005, 1997.

[5] S. H. Brongersma, E. Richard, I. Vervoort, H. Bender, W. Vandervorst, S. Lagrange, G. Beyer, and K. Maex, "Two-step room temperature grain growth in electroplated copper," Journal of Applied Physics, vol. 86, p. 3642, 1999.

[6] J. M. E. Harper, C. Cabral Jr, P. C. Andricacos, L. Gignac, I. C. Noyan, K. P. Rodbell, and C. K. Hu, "Mechanisms for microstructure evolution in electroplated copper thin films near room temperature," Journal of Applied Physics, vol. 86, pp. 2516-2525, 1999.

[7] C. Lingk and M. E. Gross, "Recrystallization kinetics of electroplated $\mathrm{Cu}$ in damascene trenches at room temperature," Journal of Applied Physics, vol. 84, pp. 5547-5553, Nov 1998.

[8] K. Pantleon and M. A. J. Somers, "In situ investigation of the microstructure evolution in nanocrystalline copper electrodeposits at room temperature," Journal of Applied Physics, vol. 100, Dec 2006.

[9] W. Wu, D. Ernur, S. H. Brongersma, M. Van Hove, and K. Maex, "Grain growth in copper interconnect lines," Microelectronic Engineering, vol. 76, pp. 190-194, Oct 2004.

[10] S. P. Hau-Riege and C. V. Thompson, "In situ transmission electron microscope studies of the kinetics of abnormal grain growth in electroplated copper films," Applied Physics Letters, vol. 76, pp. 309-311, Jan 2000.

[11] Q. T. Jiang and M. E. Thomas, "Recrystallization effects in $\mathrm{Cu}$ electrodeposits used in fine line damascene structures," Journal of Vacuum Science \& Technology B: Microelectronics and Nanometer Structures, vol. 19, pp. 762-766, 2001.

[12] D. Kwon, H. Park, S. Ghosh, C. M. Lee, H. T. Jeon, and J. G. Lee, "Recrystallization of the copper films deposited by pulsed electroplating on ECR plasma-cleaned copper seed layers," Journal of the Korean Physical Society, vol. 44, pp. 1108-1112, May 2004.

[13] S. Lagrange, S. H. Brongersma, M. Judelewicz, A. Saerens, I. Vervoort, E. Richard, R. Palmans, and K. Maex, "Self-annealing characterization of electroplated copper films," Microelectronic Engineering, vol. 50, pp. 449-457, Jan 2000.

[14] M. T. Pérez-Prado and J. J. Vlassak, "Microstructural evolution in electroplated $\mathrm{Cu}$ thin films," Journal of Applied Physics, vol. 47, pp. 817-823, 2002.

[15] C. H. Seah, G. Z. You, C. Y. Li, and R. Kumar, "Characterization of electroplated copper films for three-dimensional advanced packaging," Journal of Vacuum Science \& Technology B, vol. 22, pp. 1108-1113, May-Jun 2004. 
[16] M. Stangl, M. Lipták, A. Fletcher, J. Acker, J. Thomas, H. Wendrock, S. Oswald, and K. Wetzig, "Influence of initial microstructure and impurities on $\mathrm{Cu}$ room-temperature recrystallization (self-annealing)," Microelectronic Engineering, vol. 85 , pp. 534-541, 2008

[17] W. H. Teh, L. T. Koh, S. M. Chen, J. Xie, C. Y. Li, and P. D. Foo, "Study of microstructure and resistivity evolution for electroplated copper films at near-room temperature," Microelectronics Journal, vol. 32 , pp. $579-585,2001$

[18] V. Weihnacht and W. Brückner, "Abnormal grain growth in $\{111\}$ textured $\mathrm{Cu}$ thin films," Journal of Applied Physics, vol. 418, pp. $136-144,2002$

[19] D. W. Gan, P. S. Ho, R. Huang, J. Leu, J. Maiz, and T. Scherban, "Isothermal stress relaxation in electroplated $\mathrm{Cu}$ films. I. Mass transport measurements," Journal of Applied Physics, vol. 97, pp. 103531-1-103531-8, May 2005.

[20] K. B. Yin, Y. D. Xia, C. Y. Chan, W. Q. Zhang, Q. J. Wang, X. N. Zhao, A. D. Li, Z. G. Liu, M. W. Bayes, and K. W. Yee, "The kinetics and mechanism of room-temperature microstructura evolution in electroplated copper foils," Scripta Materialia, vol. 58, pp. $65-68,2008$

[21] K. B. Yin, Y. D. Xia, W. Q. Zhang, Q. J. Wang, X. N. Zhao, A. D. Li, Z. G. Liu, X. P. Hao, L. Wei, C. Y. Chan, K. L. Cheung, M. W. Bayes, and K. W. Yee, "Room-temperature microstructural evolution of electroplated $\mathrm{Cu}$ studied by focused ion beam and positron annihilation lifetime spectroscopy," Journal of Applied Physics, vol. 103, pp. 066103-3, 2008.

[22] M. Stangl and M. Militzer, "Modeling self-annealing kinetics in electroplated $\mathrm{Cu}$ thin films," in JOURNAL OF APPLIED PHYSICS vol. 103: AIP, 2008, p. 113521

[23] W. Zhang, S. H. Brongersma, Z. Li, D. Li, O. Richard, and K. Maex, "Analysis of the size effect in electroplated fine copper wires and a realistic assessment to model copper resistivity." vol. 101: Journal of applied physics, 2007, p. 063703.

[24] W. Robl, M. Melzl, B. Weidgans, R. Hofmann, and M. Stecher, "Last Metal Copper Metallization for Power Devices," in IEEE Transactions on Semiconductor Manufacturing, 2008, pp. 358-362.

[25] M. F. Doerner and W. D. Nix, "Stresses and deformation processes in thin films on substrates," Critical Reviews in Solid State and Materials Sciences, vol. 14, pp. 225-268, 1988.

[26] L. B. Freund and S. Suresh, Thin Film Materials: Stress, Defect Formation, and Surface Evolution: Cambridge University Press, 2003.

[27] W. D. Nix, "Mechanical Properties of Thin Films," Metallurgical Transactions A, vol. 20, pp. 2217-2245, 1989 .

[28] C. Detavernier, S. Rossnagel, C. Noyan, S. Guha, C. Cabral, and C. Lavoie, "Thermodynamics and kinetics of room-temperature microstructural evolution in copper films," Journal of Applied Physics, vol. 94, pp. 2874-2881, Sep 2003.

[29] P. Chaudhari, "Grain Growth and Stress Relief in Thin Films," in Journal of Vacuum Science and Technology. vol. 9, 1972, pp. $520-522$

[30] T. H. Courtney and T. Hugh, Mechanical behavior of materials. New York McGraw-Hill New York, 1990.

[31] R. M. Keller, S. P. Baker, and E. Arzt, "Quantitative analysis of strengthening mechanisms in thin $\mathrm{Cu}$ films: Effects of film thickness, grain size, and passivation," in Journal of Materials Research. vol. 13: Pittsburgh, PA: Published for the Materials Research Society by the American Institute of Physics, c1986-, 1998, pp. 1307-1317.

[32] R. Venkatraman and J. C. Bravman, "Separation of film thickness and grain boundary strengthening effects in $\mathrm{Al}$ thin films on $\mathrm{Si}$," in Journal of Materials Research. vol. 7, 1992, pp. 2040-2048.

[33] H. Lee, S. S. Wong, and S. D. Lopatin, "Correlation of stress and texture evolution during self- and thermal annealing of electroplated Cu films," Journal of Applied Physics, vol. 93, pp. 3796-3804, Apr 2003.

[34] C. Cabral, P. C. Andricacos, L. Gignac, I. C. Noyan, K. P. Rodbell, T. M. Shaw, R. Rosenberg, J. M. E. Harper, P. W. DeHaven, and P. S. Locke, "Room temperature evolution of microstructure and resistivity in electroplated copper films," in Advanced Metallization Conference, Colorado, 1998, p. 81. 